\title{
Azimuthal expansion of the dipolarization at geosynchronous orbits associated with substorms
}

\author{
C. J. Watson and P. T. Jayachandran \\ Physics Dept., University of New Brunswick, P.O. Box 4400, 8 Bailey Drive, Fredericton, New Brunswick, E3B 5A3, Canada
}

Received: 29 September 2008 - Revised: 16 February 2009 - Accepted: 16 February 2009 - Published: 5 March 2009

\begin{abstract}
Dipolarization, one of the main indicators of substorm expansion onset, represents topological changes in the magnetotail. It is believed that dipolarization is initiated at a longitudinally confined region in the tail, followed by the azimuthal expansion. There are very limited studies in the literature regarding the azimuthal propagation of the dipolarization front in the magnetotail. In this study we have used ten years of GOES data and POLAR and IMAGE data to study the characteristics of the propagation of the dipolarization fronts at the geosynchronous orbit. We have identified a number of dipolarization events in the GOES magnetic field data and substorm onsets from POLAR UVI and IMAGE-FUV measurements. Using the delay of dipolarization signatures at the two GOES satellites and onset times from POLAR and IMAGE measurements we have estimated the propagation speed of the dipolarization fronts. The calculated speeds vary between $10 \mathrm{~km} / \mathrm{s}$ and $420 \mathrm{~km} / \mathrm{s}$ and show a power law distribution.
\end{abstract}

Keywords. Magnetospheric physics (Auroral phenomena; Magnetosphere-ionosphere interactions; Magnetospheric configuration and dynamics)

\section{Introduction}

Dipolarization in the near Earth magnetotail has become recognized as a signature of magnetospheric substorms (Belehaki et al., 1998; Frank et al., 2000; Liou et al., 2002; Jayachandran et al., 2007) and its study can be critical in understanding substorm dynamics and the mechanism behind substorm onset. Dipolarization is a topological change of the inner magnetosphere from a stretched (tail-like) configuration to a relaxed (dipole-like) magnetic field configura-

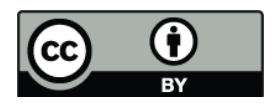

Correspondence to: C. J. Watson (chris.watson@unb.ca) tion. Liou et al. (2001) have shown that there exists a temporal delay between auroral onset and magnetic field dipolarization at GOES, representing the latitudinal and longitudinal propagation of the auroral bulge. Also shown is a close mapping between the ionospheric auroral bulge and dipolarization in the magnetotail. Dipolarization coinciding with substorm onset occurs initially in a narrow, localized tail region in the $6-10 R_{E}$ range. This synchronal occurrence is often explained by diversion of the cross tail current into the auroral ionosphere when energy buildup in the magnetotail reaches a critical level (McPherron et al., 1973; Lui, 1991). This current diversion forms the well known substorm current wedge, which flows through the westward electrojet in the ionosphere. Auroral arc expansion is associated with electrojet current expansion as well as expansion of the disrupted cross tail current in the neutral plasma sheet. Initial localized dipolarization due to initial current disruption is followed by an azimuthal (Kokubun and McPherron, 1981; Liou et al., 2002) and radial (Ohtani, 2001) propagation in the magnetotail associated with a poleward and equatorward expansion of the auroral arcs (Lopez and Lui, 1990).

Understanding the azimuthal expansion of the dipolarization front is crucial in understanding the substorm process altogether. One of the easiest ways to study this expansion is to look at the geosynchronous magnetic field data for signatures of dipolarization associated with substorms. As the propagating dipolarization is closely associated with azimuthal expansion of auroral arcs, one can use temporal delays between onset times identified by global auroral images such as POLAR and IMAGE to estimate the propagation speed of dipolarization fronts at geosynchronous orbits. In this study we have used GOES magnetic field data combined with POLAR and IMAGE data to study the azimuthal expansion of the dipolarization fronts at geosynchronous orbits. Between March 1996 and December 2005, several hundred substorm onsets were identified from POLAR UVI (Liou et al., 2001) (May 1996-December 1999 onsets) and IMAGE-FUV (Frey

Published by Copernicus Publications on behalf of the European Geosciences Union. 


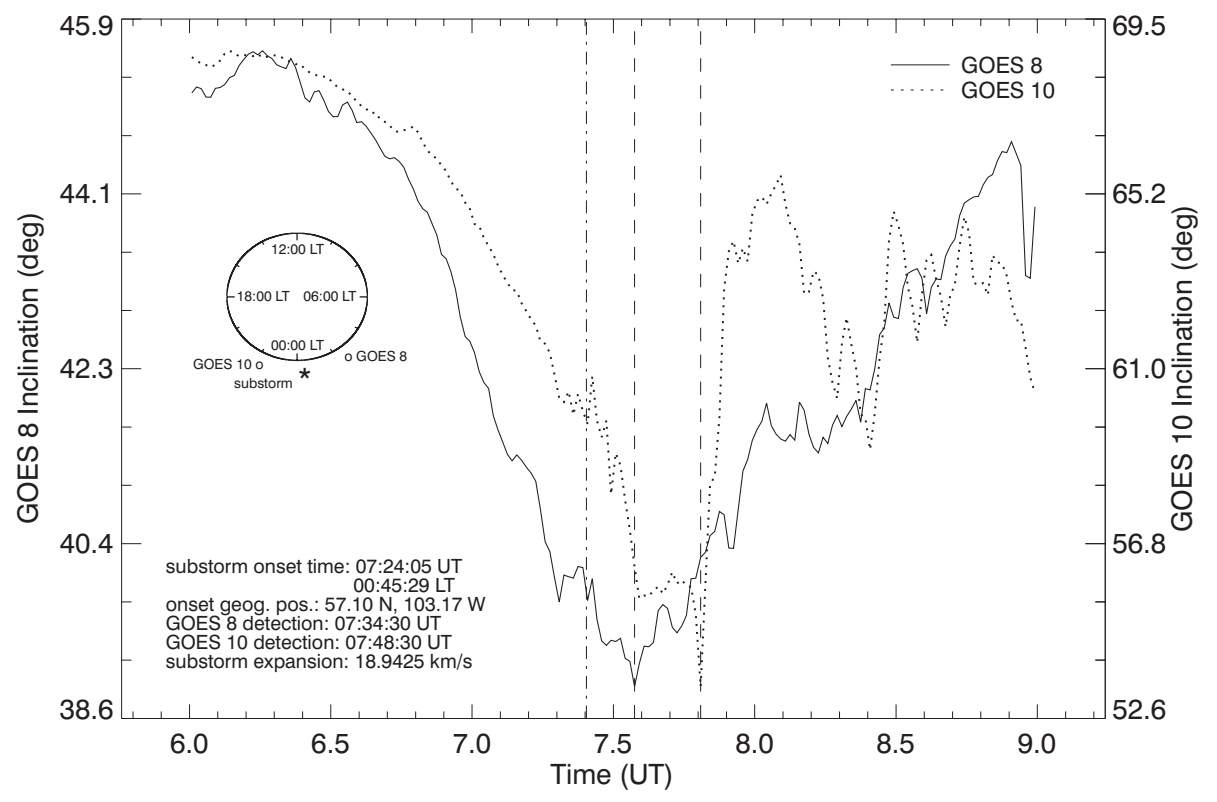

Fig. 1. Temporal variation in magnetic inclination calculated from GOES 8 and GOES 10 magnetometer data on 31 August 2000. Substorm onset is indicated by the vertical dash-dot line and GOES detection of dipolarization fronts by dashed lines at the local inclination minimum for each satellite. Also plotted is a local time dial showing local time of substorm onset relative to each satellite.

et al., 2004) (May 2000-December 2005 onsets) data. Onset latitude, longitude and time were used in conjunction with GOES data to monitor the evolution of dipolarization at the geosynchronous orbit associated with substorm events.

\section{Data, method of analysis and results}

We have used GOES magnetic field data to identify magnetic field dipolarization in the magnetotail associated with substorms. GOES data is time-averaged in 1 min bins tagged at $30 \mathrm{~s}$ passed the minute. Magnetic inclination was calculated at GOES east $\left(75^{\circ}\right.$ west $)$ and west $\left(135^{\circ}\right.$ west $)$ from magnetometer measurements, as in Eq. (1).

$I=\tan ^{-1} \frac{B_{z}}{\sqrt{B_{x}^{2}+B_{y}^{2}}}$

We have calculated inclination to avoid use of multiple parameters such as three magnetic field components. We have used substorm onsets identified by Liou et al. (2001) and Frey et al. (2004) from POLAR UVI and IMAGE satellite data. From these databases we have chosen isolated substorm onset times, latitudes and longitudes. An example of the temporal variation of magnetic field inclination around a substorm onset time is shown in Fig. 1. This substorm event of 31 August 2000 occurred with onset at 07:24:05 UT, indicated by a vertical dash-dot line in the figure. Dipolarization at each GOES followed this onset and is indicated by vertical dashed lines. Initial drop in inclination at each satellite suggests stretching of the magnetotail associated with the substorm growth phase. This is followed by an increase in inclination representing dipolarization onset at 07:34:30 UT for GOES 8 and 07:48:30 UT for GOES 10. Geographic local time of substorm onset relative to GOES local times is also indicated in the Fig. 1 insert. Onset occurs near local midnight at 00:15:48 LT, between GOES 8 (02:24:05 LT) and GOES 10 (22:25:05 LT). Temporal delay between onset time and when dipolarization signatures reach GOES satellites indicates a propagation of the dipolarization fronts. The difference in delay time of dipolarization (GOES West minus GOES East) is shown in Fig. 2, with $1 \mathrm{~h}$ time averages of these delays indicated by large, diamond symbols. This distribution is similar to that shown by Nagai (1991). By ignoring radial propagation effects and assuming equal east and west azimuthal propagations (see discussion), the azimuthal expansion speed of the dipolarization fronts can be calculated from the temporal delay between onset and dipolarization identification times.

We have limited our study to onsets occurring between 00:00-11:00 UT and $60^{\circ}-70^{\circ} \mathrm{N}$ geomagnetic latitudes to limit radial propagation of dipolarization fronts. The chosen time interval singles out onsets occurring at longitudes between GOES satellites. By limiting our onset times and latitudes as mentioned above we have identified 127 isolated substorm events for analysis. Figure 3 indicates latitude and local time distribution of onsets used in the study.

Calculating expansion speeds of dipolarization fronts for these 127 events, we found values ranging between $10 \mathrm{~km} / \mathrm{s}$ (lowest) and $420 \mathrm{~km} / \mathrm{s}$ (highest). Examples of studied events are shown in Figs. 4, 5 and 6. Figure 4 plots temporal 


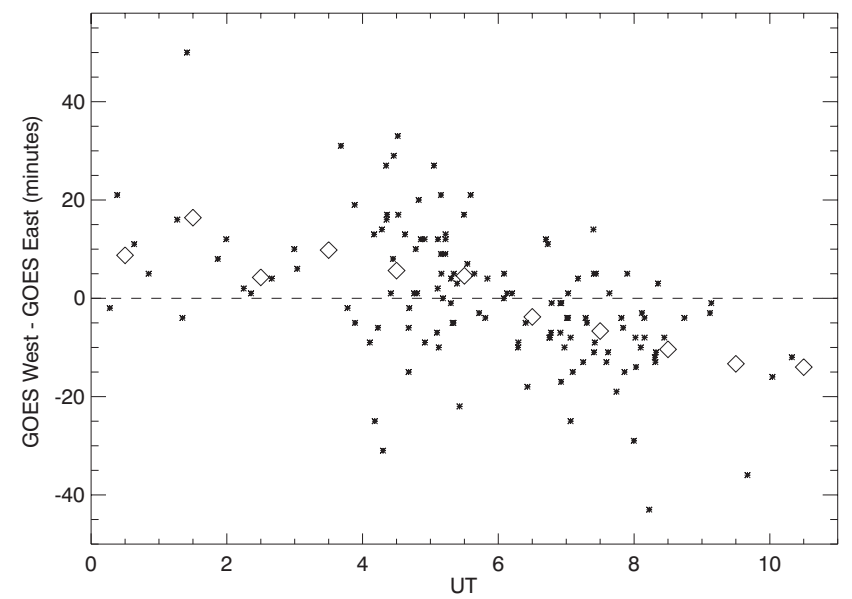

Fig. 2. Delay in local dipolarization onset between GOES East (GOES 8 or 12) and GOES west (GOES 9 or 10) for substorm onset times. The large diamond shapes indicate $1 \mathrm{~h}$ averages of these delays.

variation in inclination on 20 February 2001 with substorm onset at 10:19:32 UT, followed by dipolarization at GOES 10 at 10:32:30 and GOES 8 at 10:44:30, giving an expansion speed of $19.5 \mathrm{~km} / \mathrm{s}$. This speed was quite typical for this study and represents the range in which most speeds occurred. Figure 5 shows magnetic inclination for a 18 July 2001 substorm. We found temporal delays of 1 and $2 \mathrm{~min}$ between onset and dipolarizations for GOES 8 and 10 for this event, giving an expansion speed of $223.3 \mathrm{~km} / \mathrm{s}$. This event type was rare as few speeds fell above $100 \mathrm{~km} / \mathrm{s}$. The highest calculated speed was $419.8 \mathrm{~km} / \mathrm{s}$ for the event represented in Fig. 6. Delay time was $20 \mathrm{~s}$ for GOES 8 and $80 \mathrm{~s}$ for GOES 10 (20 s delay is estimated from the $1 \mathrm{~min}$ average GOES data, therefore the delay is ambiguous by $1 \mathrm{~min}$ ). A histogram showing the distribution of calculated speeds is shown in Fig. 7. A large number of propagations occur in the 20 to $60 \mathrm{~km} / \mathrm{s}$ range with the number of events dropping as speed increases indicating a power law probability distribution.

\section{Discussion}

We have used GOES magnetic field measurements along with POLAR UVI and IMAGE-FUV data to estimate the azimuthal expansion speed of dipolarization fronts at the geosynchronous orbits. Estimated speeds varied between $\sim 10 \mathrm{~km} / \mathrm{s}$ (lowest) and $\sim 420 \mathrm{~km} / \mathrm{s}$ (highest) with most speeds in the $20-60 \mathrm{~km} / \mathrm{s}$ range. This result confirms earlier studies that dipolarization initially occurs in a narrowly confined longitudinal region (Kokubun and McPherron, 1981; Liou et al., 2002). Propagation speeds are similar to those reported by Liou et al. (2002). The results presented in this paper related to the expansion speed may be ambiguous for

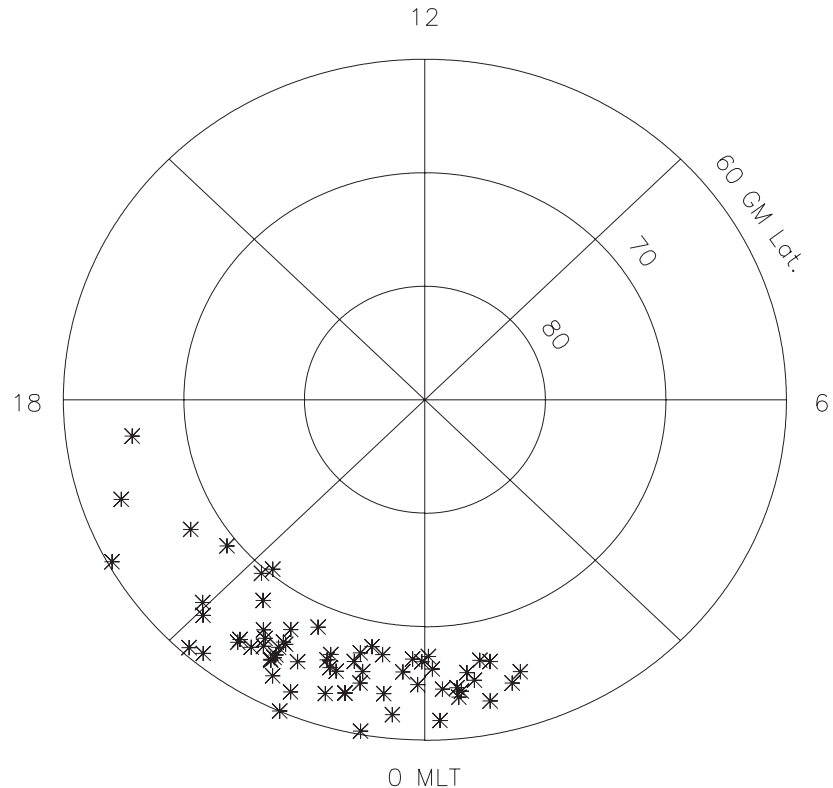

Fig. 3. Geomagnetic latitudes and magnetic local times of some substorm onsets used in this study.

the following reasons: 1). Assumption that Eastward and Westward propagation speeds are the same. We have separately estimated the Eastward and Westward propagation speeds and found an average Westward speed of $\sim 51 \mathrm{~km} / \mathrm{s}$ and average Eastward speed of $\sim 56 \mathrm{~km} / \mathrm{s}$. The difference in propagation speeds is small and justifies our assumption. Moreover, earlier studies (e.g. Liou et al., 2002) reported only slight variation in the Eastward and Westward speeds of propagation. Figure 2 also supports this assumption since dipolarization delays between GOES East and West are similar for pre-midnight (00:00-05:00 UT) and post-midnight (09:00-11:00 UT). We found a small number of events in which Eastward and Westward speeds were significantly different. 2). Neglecting the radial propagation effects. We have limited our study to substorm onsets occurring near the geosynchronous orbit to minimize radial propagation effects. We know that this justification is questionable, and it can be addressed and resolved only using THEMIS along with GOES data. A study pertaining to this matter is currently in progress. The distribution of speeds (Fig. 7) is a broad distribution, which logically gives rise to the question of what controls the speed of expansion. In an attempt to find a solution we have compared the speed with different internal tail parameters such as magnetic field strength and gradient of the $\boldsymbol{B}$ field using GOES measurements, but could not find any meaningful correlations. We also compared speed with external driver parameters such as Interplanetary Magnetic Field (IMF) and Solar Wind and again did not find any correlations. Two parameters that we did not investigate are plasma sheet density and plasma pressure due to lack of available data. 


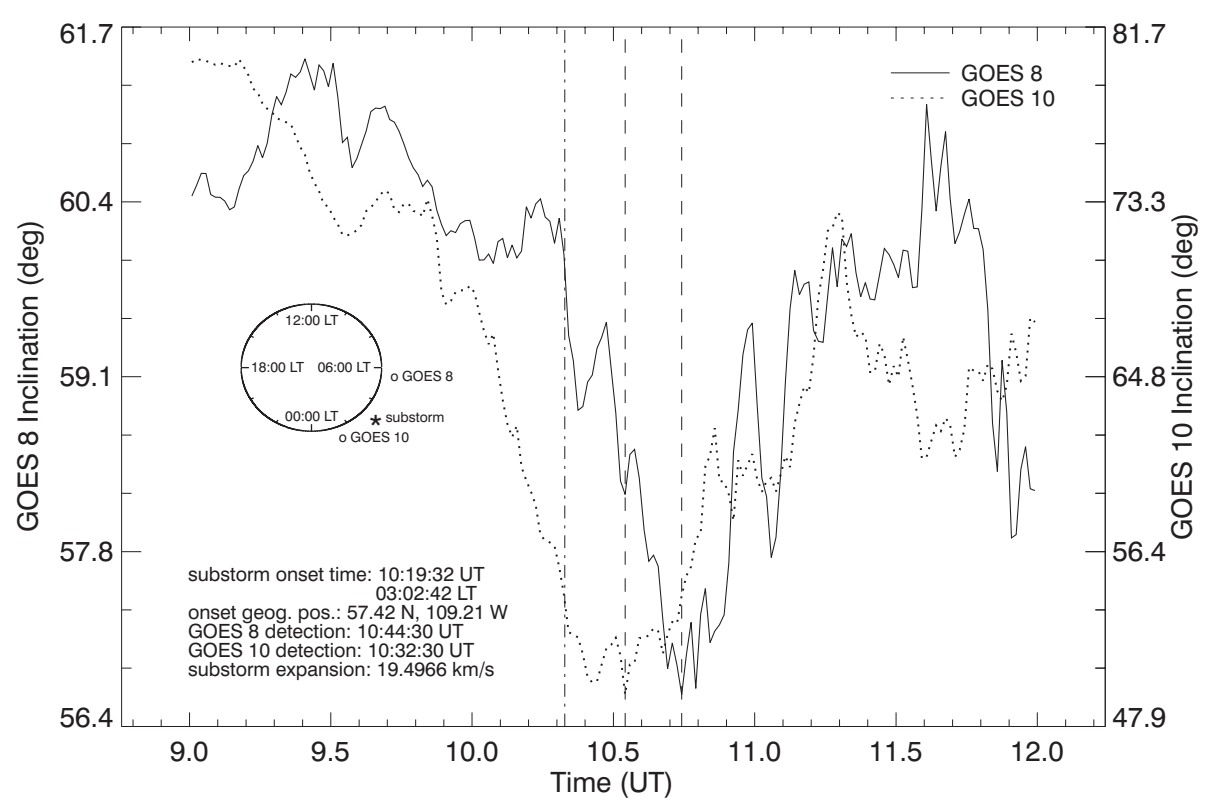

Fig. 4. Magnetic inclination calculated from GOES data showing dipolarization signatures of a substorm event on 20 February 2001.

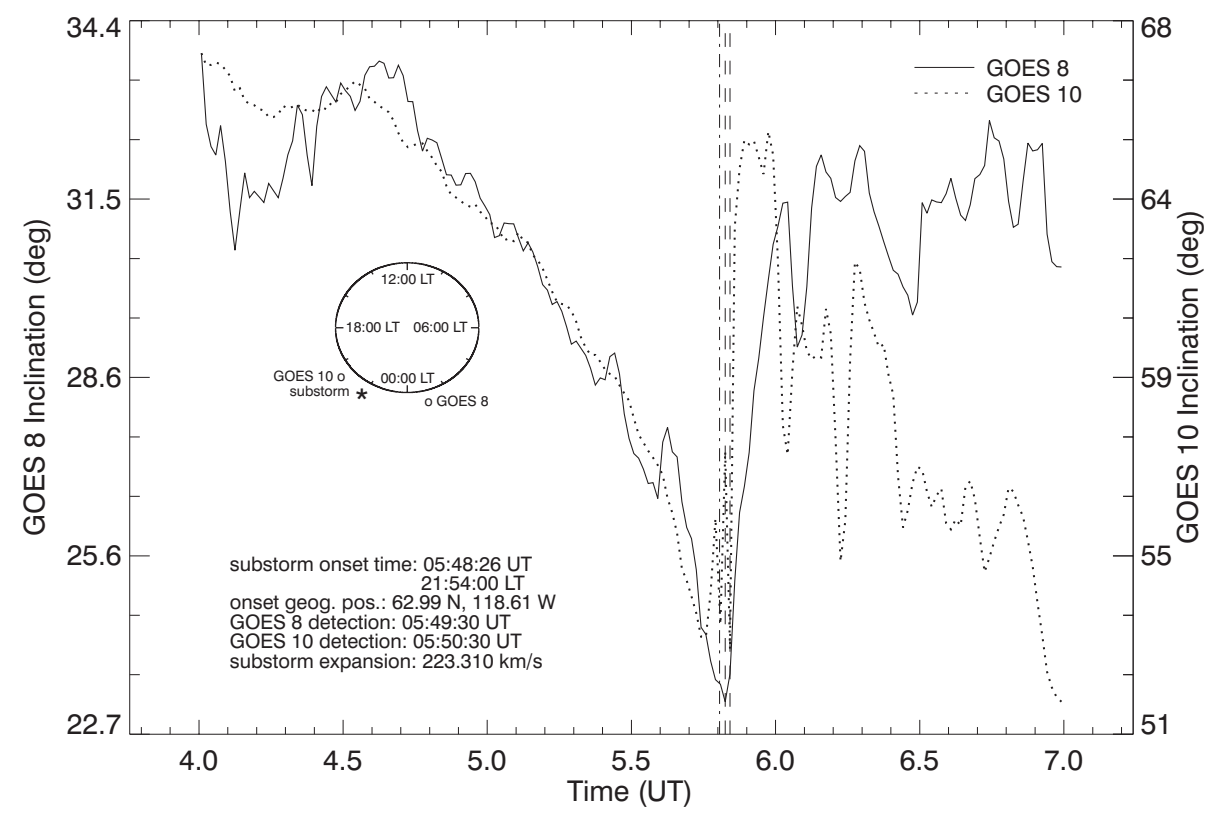

Fig. 5. Magnetic inclination calculated from GOES data showing dipolarization signatures of a substorm event on 18 July 2001.

It is interesting to note that previous studies (e.g. Lui et al., 2000) of some parameters associated with substorms have revealed similar distributions to the one shown in Fig. 7. To determine the power law coefficient of the propagation speed distribution, a log-log plot of this distribution was produced (Fig. 8) with a best-fit line over selected data. We find the propagation speed follows a power law with index -1.256 . This finding is consistent with Lui et al. (2000), who reported power law distributions in auroral blob size and intensity with indices of -1.206 and -1.049 , respectively, for active substorm times. The same research reported similar power law coefficients for quiet times indicating a dissipation of energy independent of magnetospheric activity. This property indicates a system operating under the sandpile model (Bak et al., 1987) in a self-organized critical (SOC) state. In a system governed by this model there exists a threshold or critical state that when exceeded triggers an "avalanche", or event characteristic of the system, to relieve "pressure" and 


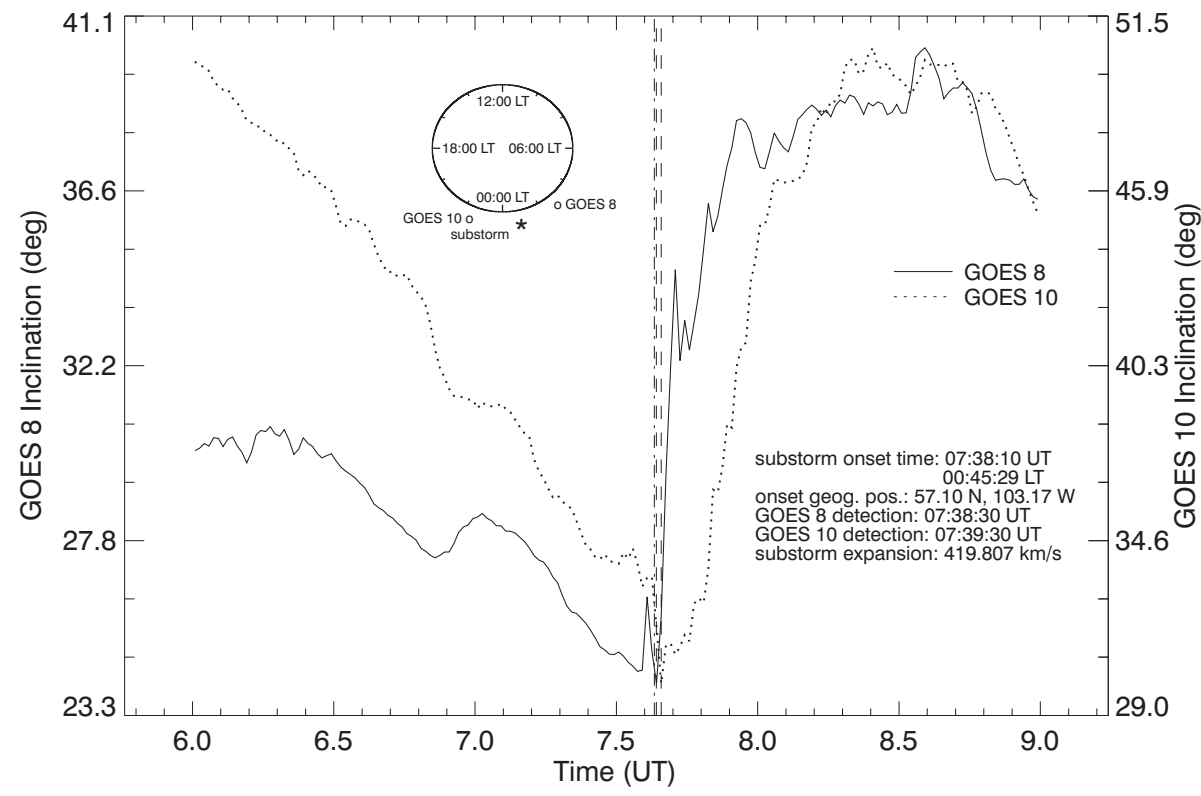

Fig. 6. Magnetic inclination calculated from GOES data showing dipolarization signatures of a substorm event on 8 May 2001 .

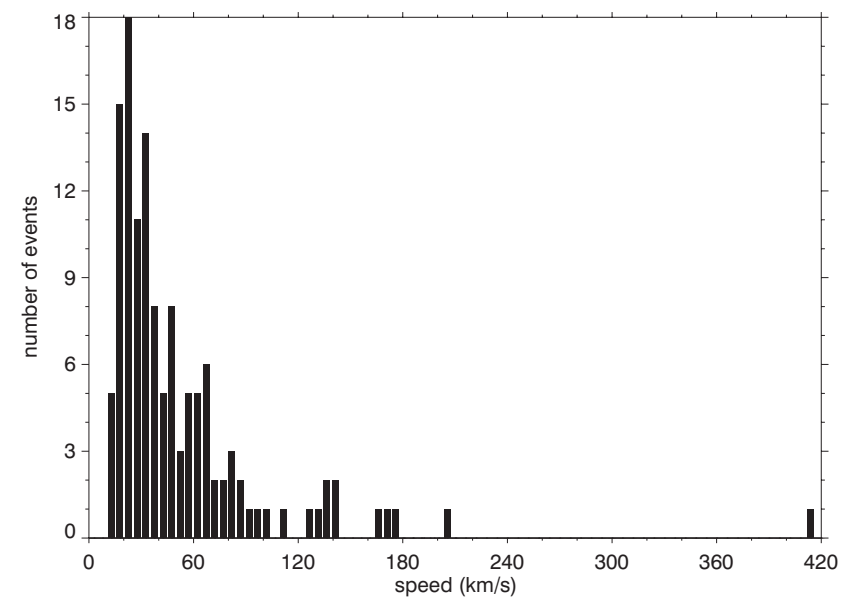

Fig. 7. Distribution of azimuthal expansion speeds of dipolarization fronts.

prevent it from exceeding that critical state. The parameters of the event are dependent on the internal state of the system and independent of external variables. In the case of substorms it is proposed that energy build up in the magnetotail reaches a critical state triggering an "avalanche" or release of energy via cross tail current diversion. The amount of energy release follows a power law probability distribution and determines the magnitude of the substorm event.
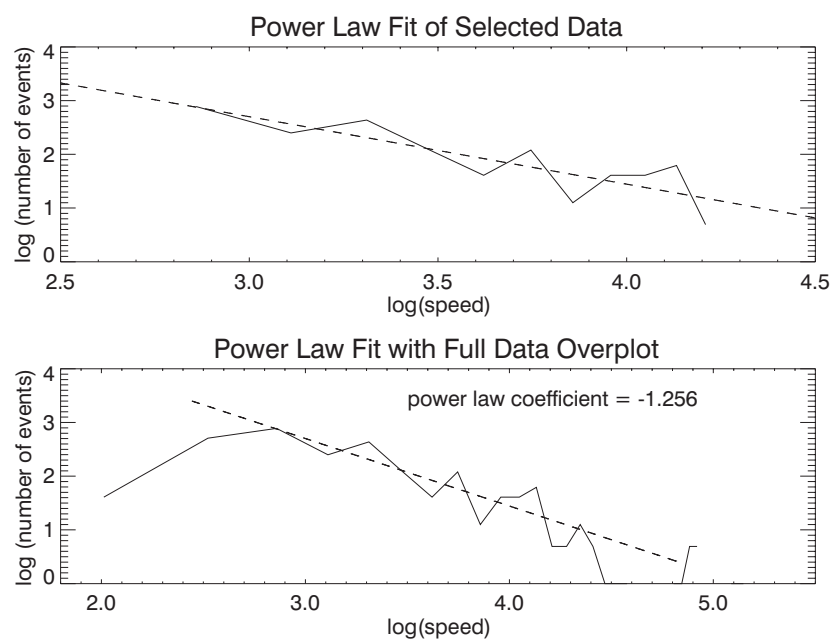

Fig. 8. Log-log plot of the speed distribution in Fig. 7. Distribution follows a power law with an index of -1.256 .

\section{Conclusions}

Sharp drops then sudden increases in magnetic inclination, as calculated from GOES data, were found to occur around substorm onset times reconfirming dipolarization of the Earth's magnetic field as a signature of substorm onset. Temporal delays between onset times observed by POLAR and IMAGE data and correlating dipolarization events determined from GOES data indicate azimuthally propagating dipolarization fronts with speeds varying between $10 \mathrm{~km} / \mathrm{s}$ and $420 \mathrm{~km} / \mathrm{s}$. In order to unambiguously determine the speed we require data from satellite constellations such as THEMIS. A 
study is underway using THEMIS data in conjunction with GOES data. However, results presented in this study give us a cursory idea of substorm expansion dynamics at geosynchronous orbits. Propagation speeds follow a power law with an index of -1.256 . This is similar to previous findings in which power law distributions for auroral size and intensity are reported. Findings support the "sandpile" or "avalanche" model for substorm dynamics, in which event parameters are governed by self-organized criticality.

Acknowledgements. This research at UNB was supported by the Natural Science and Engineering Research Council (NSERC) of Canada.

Topical Editor R. Nakamura thanks two anonymous referees for their help in evaluating this paper.

\section{References}

Bak, P., Tang, C., and Wiesenfeld, K.: Self-organized criticality: An explanation of 1/f noise, Phys. Rev. Lett., 59, 381-384, 1987.

Belehaki, A., Tsagouri, I., and Mavromichalaki, H.: Study of the longitudinal expansion velocity of the substorm current wedge, Ann. Geophys., 16, 1423-1433, 1998, http://www.ann-geophys.net/16/1423/1998/.

Cheng, C. Z.: Physics of substorm growth phase, onset and dipolarization, Space Sci. Rev., 113, 207-270, 2004.

Frank, L. A., Paterson, W. R., Sigwarth, J. B., and Kokubun, S.: Observations of magnetic field dipolarization during auroral substorm onset, J. Geophys. Res., 105, 15897-15912, 2000.

Frey, H. U., Mende, S. B., Angelopoulus, V., and Donovan, E. F.: Substorm onset observations by IMAGE-FUV, J. Geophys. Res., 109, 10304.1-10304.6, 2004.
Jayachandran, P. T., MacDougall, J. W., Hamza, A. M., and Henderson, M. G.: Observations of dipolarization at geosynchronous orbits and its response in the polar cap convection during extreme southward interplanetary magnetic field conditions, J. Geophys. Res., 112, 10216.1-10216.5, 2007.

Kokubun, S. and McPherron, R. L.: Substorm signatures at synchronous altitude, J. Geophys. Res., 86, 11265-11277, 1981.

Liou, K., Meng, C. I., Lui, A. T. Y., Newell, P. T., and Wing, S.: Magnetic dipolarization with substorm expansion onset, J. Geophys. Res., 107, 1131, doi:10.1029/2001JA000179, 2002.

Liou, K., Newell, P. T., Sibeck, D. G., Meng, C. I., Brittnacher, M., and Parks, G.: Observation of IMF and seasonal effects in the location of auroral substorm onset, J. Geophys. Res., 106, 5799_ 5810, 2001.

Lopez, R. E. and Lui, A. T. Y.: A multisatellite case study of the expansion of a substorm current wedge in the near-earth magnetotail, J. Geophys. Res., 95, 8009-8017, 1990.

Lui, A. T. Y., Chapman, S. C., Liou, K., Newell, P. T., Meng, C. I., Brittnacher, M., and Parks, G.K.: Is the dynamic magnetosphere an avalanching system?, Geophys. Res. Lett., 27, 911-914, 2000.

Lui, A. T. Y.: A synthesis of magnetospheric substorm models, J. Geophys. Res., 96, 1849-1856, 1991.

McPherron, R. L., Russell, C. T., and Aubry, M. P.: Satellite studies of magnetospheric substorms on August 15, 1968, Phenomenological model for substorms, J. Geophys. Res., 78, 3131-3149, 1973.

Nagai, T.: An empirical model of substorm-related magnetic field variations at synchronous orbit, Geophys. Monogr. Ser., 64, 9195, 1991.

Ohtani, S.: Substorm trigger processes in the magnetotail: recent observations and outstanding issues, Space Sci. Rev., 95, 347359, 2001. 